\title{
Theoretical Study on Fe-Se-H Hydrides under High Pressure
}

Jiayao Chen ${ }^{\dagger}$, Xuyan Xue $e^{\dagger}$, Hailiang Chen ${ }^{\dagger}$, Haifeng Liù ${ }^{\dagger}$, Qingjun Zang ${ }^{\dagger}$ and Wencai $L u^{*,+, t}$

${ }^{\dagger}$ College of Physics and State Key Laboratory of Biological Polysaccharide Fiber Forming and Ecological Textile, State Key Laboratory, Qingdao University, Qingdao, 266071, P. R. China

Institute of Theoretical Chemistry, Jilin University, Jilin 130021, P. R. China 

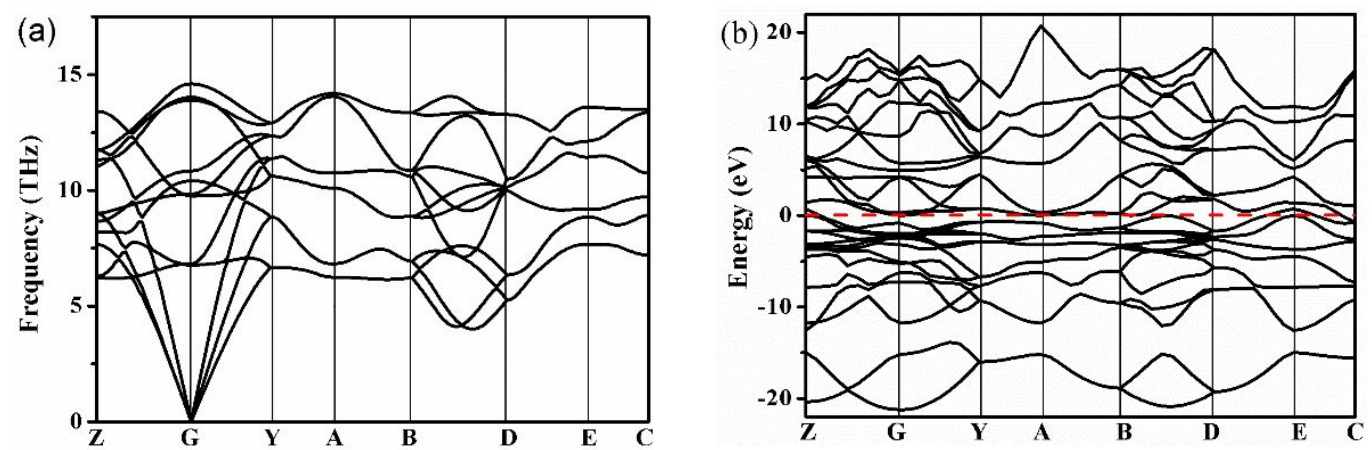

Figure S1. (a) Phonon dispersion curves and (b) electronic band structure of $\mathrm{R} \overline{3}$ $\mathrm{m}-\mathrm{FeSe}$ phase at $150 \mathrm{GPa}$. 

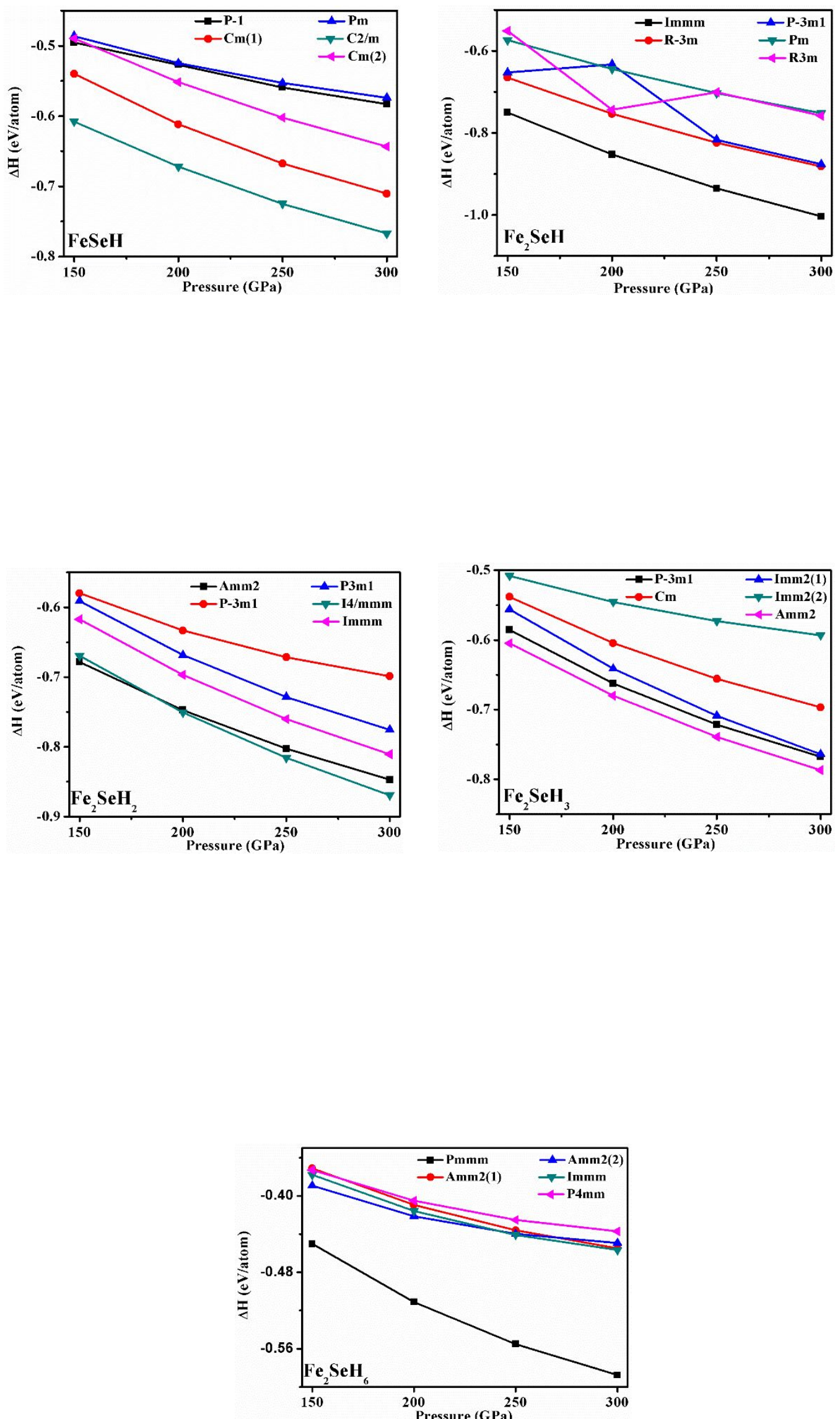
Figure $\mathrm{S} 2$. Formation enthalpies $(\Delta \mathrm{H}$ in $\mathrm{eV} /$ atom) with respect to $\mathrm{Fe}, \mathrm{Se}$ and $\mathrm{H}$ for the $\mathrm{FeSeH}, \mathrm{Fe}_{2} \mathrm{SeH}, \mathrm{Fe}_{2} \mathrm{SeH}_{2}, \mathrm{Fe}_{2} \mathrm{SeH}_{3}$ and $\mathrm{Fe}_{2} \mathrm{SeH}_{6}$ phases at 150, 200, 250 and $300 \mathrm{GPa}$, respectively. 

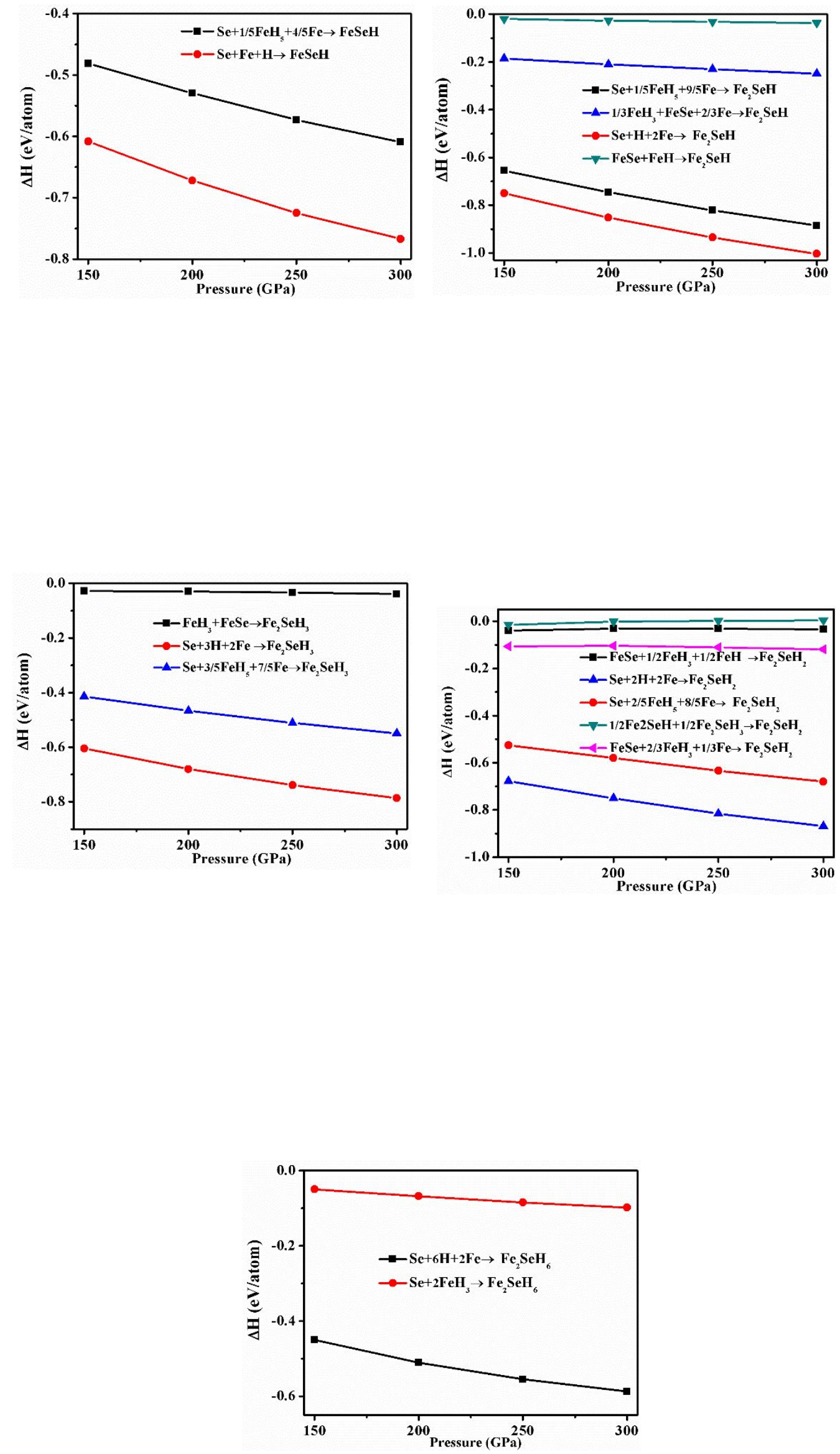
Figure S3. Different synthetic pathways of the $\mathrm{FeSeH}, \mathrm{Fe}_{2} \mathrm{SeH}, \mathrm{Fe}_{2} \mathrm{SeH}_{2}, \mathrm{Fe}_{2} \mathrm{SeH}_{3}$ and $\mathrm{Fe}_{2} \mathrm{SeH}_{6}$ phases at 150, 200, 250 and $300 \mathrm{GPa}$, respectively. 


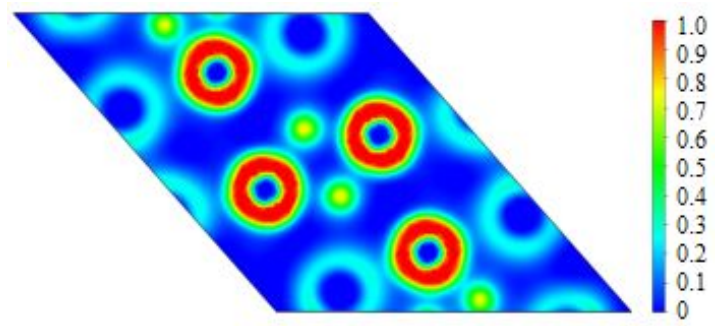

(a)

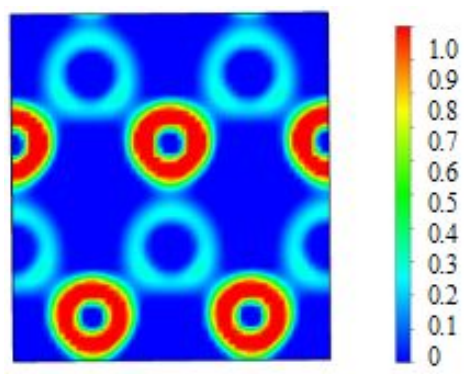

(c)

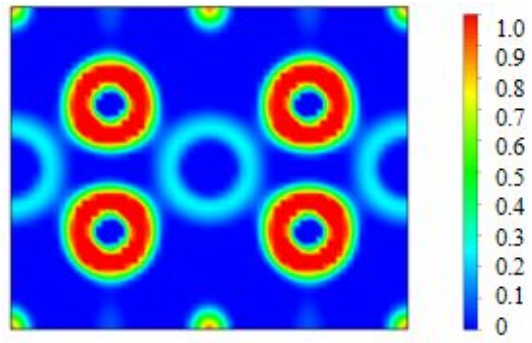

(b)

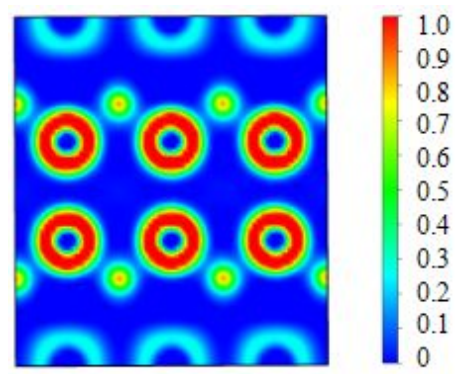

(d)

Figure S4. Electron localization function (ELF) maps of (a) $\mathrm{C} 2 / \mathrm{m}-\mathrm{FeSeH}$, (b) Immm- $\mathrm{Fe}_{2} \mathrm{SeH}$, (c) Amm2- $\mathrm{Fe}_{2} \mathrm{SeH}_{2}$, (d) $\mathrm{I} 4 / \mathrm{mmm}-\mathrm{Fe}_{2} \mathrm{SeH}_{2}$, (e) $\mathrm{Amm} 2-\mathrm{Fe}_{2} \mathrm{SeH}_{3}$ and (f)

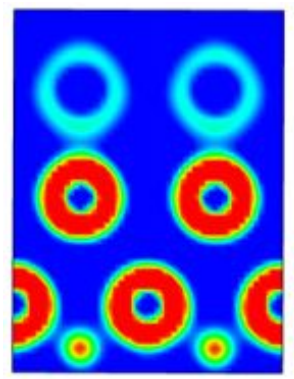

(e)

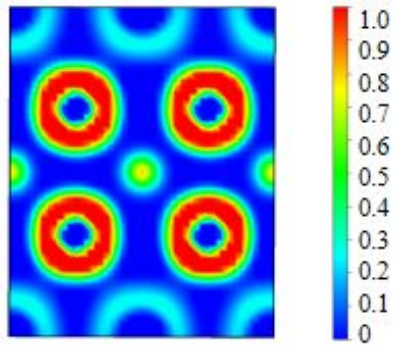

(f)

Pmmm- $\mathrm{Fe}_{2} \mathrm{SeH}_{6}$ phases at $150 \mathrm{GPa}$. 


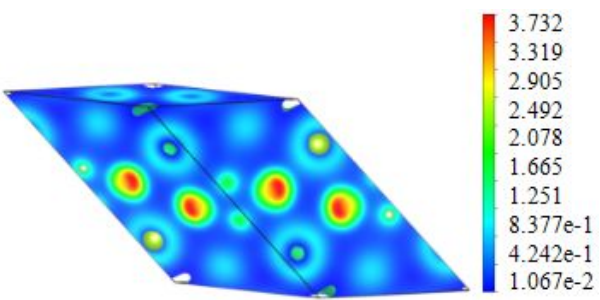

(a)

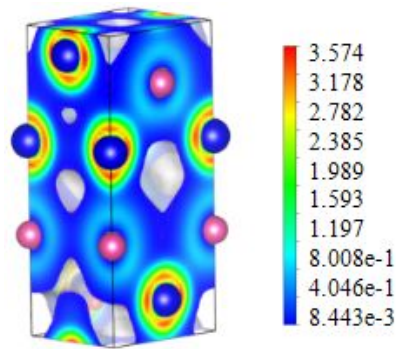

(c)

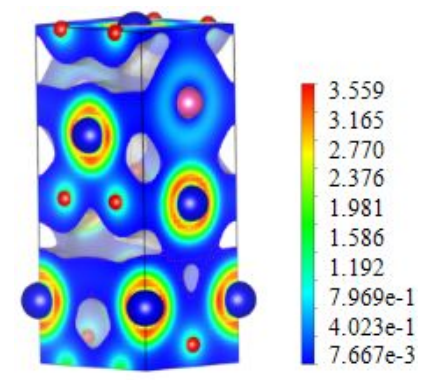

(e)

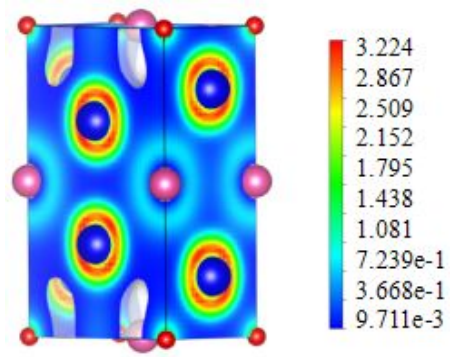

(b)

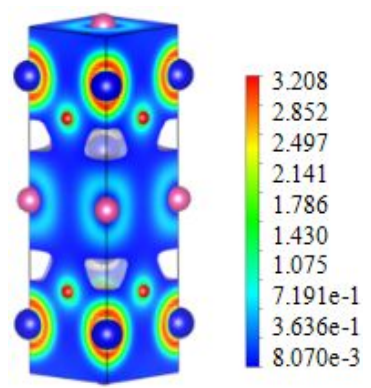

(d)

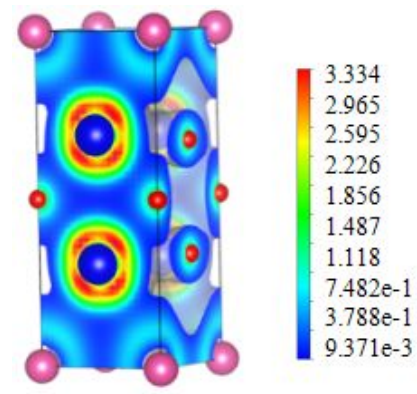

(f) 
Figure S5. Electron densities of (a) $\mathrm{C} 2 / \mathrm{m}-\mathrm{FeSeH}$, (b) $\mathrm{Immm}-\mathrm{Fe}_{2} \mathrm{SeH}$, (c)

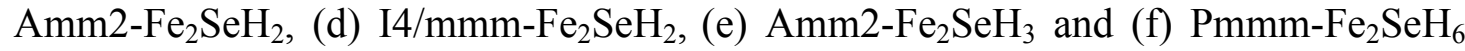
phases at $150 \mathrm{GPa}$. Blue, red and pink spheres denote $\mathrm{Fe}, \mathrm{H}$ and $\mathrm{Se}$ atoms, respectively. 


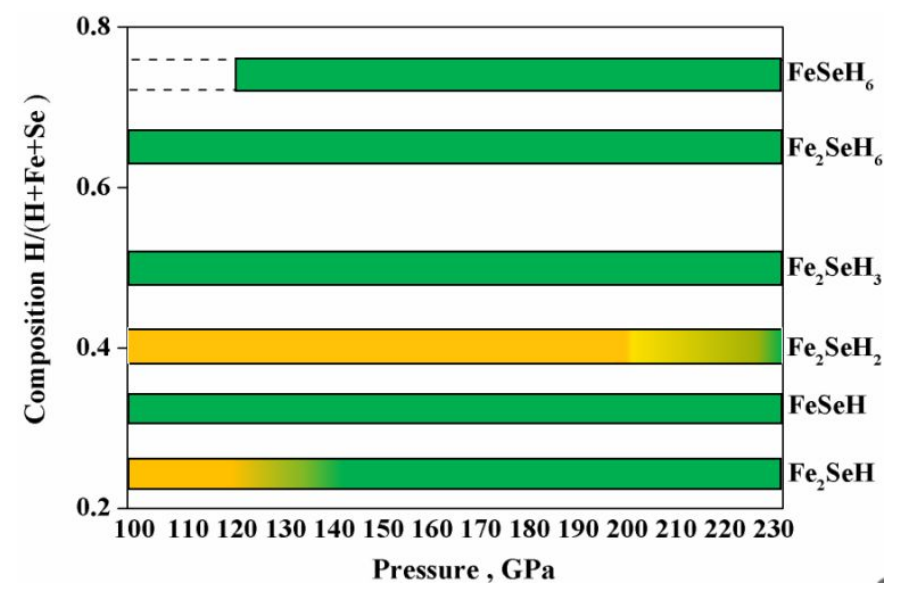

Figure S6. Pressure-composition phase diagram of the $\mathrm{C} 2 / \mathrm{m}-\mathrm{FeSeH}, \mathrm{Immm}-\mathrm{Fe}_{2} \mathrm{SeH}$, $\mathrm{I} 4 / \mathrm{mmm}_{-} \mathrm{Fe}_{2} \mathrm{SeH}_{2}, \mathrm{Amm} 2-\mathrm{Fe}_{2} \mathrm{SeH}_{3} \mathrm{Pm}-\mathrm{FeSeH}_{6}$ and $\mathrm{Pmmm}-\mathrm{Fe}_{2} \mathrm{SeH}_{6}$ phases. Green indicates having no magnetism and yellow represents being magnetic.

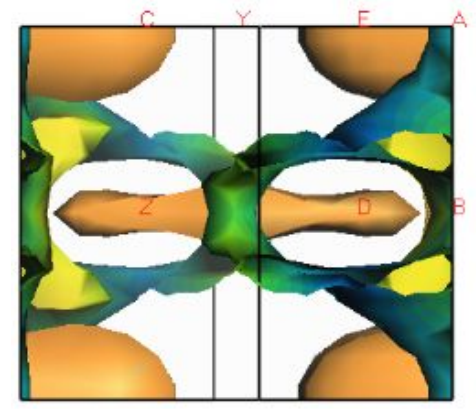

(a)

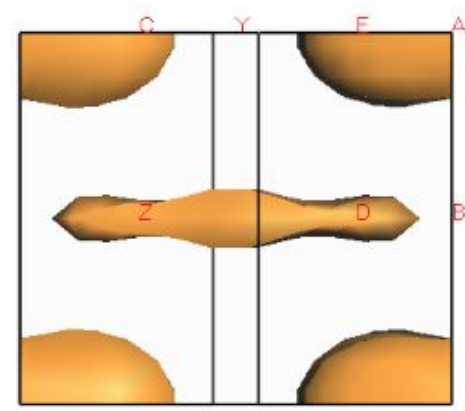

(c)

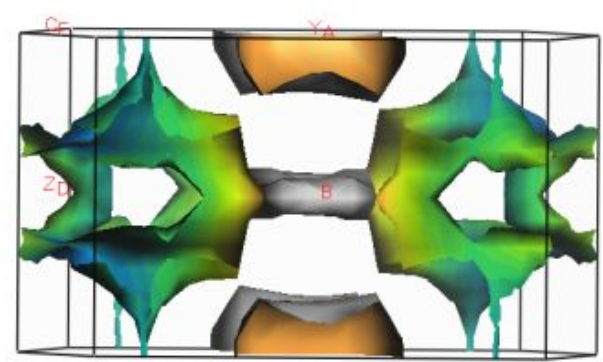

(b)

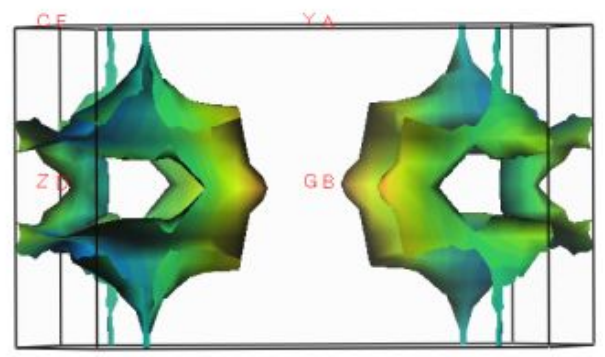

(d) 
Figure S7. (a) and (b) Fermi surface, and (c) and (d) decomposed fermi surface in the Brillouin zone of the $\mathrm{Pm}-\mathrm{FeSeH}_{6}$ phase at $150 \mathrm{GPa}$. 

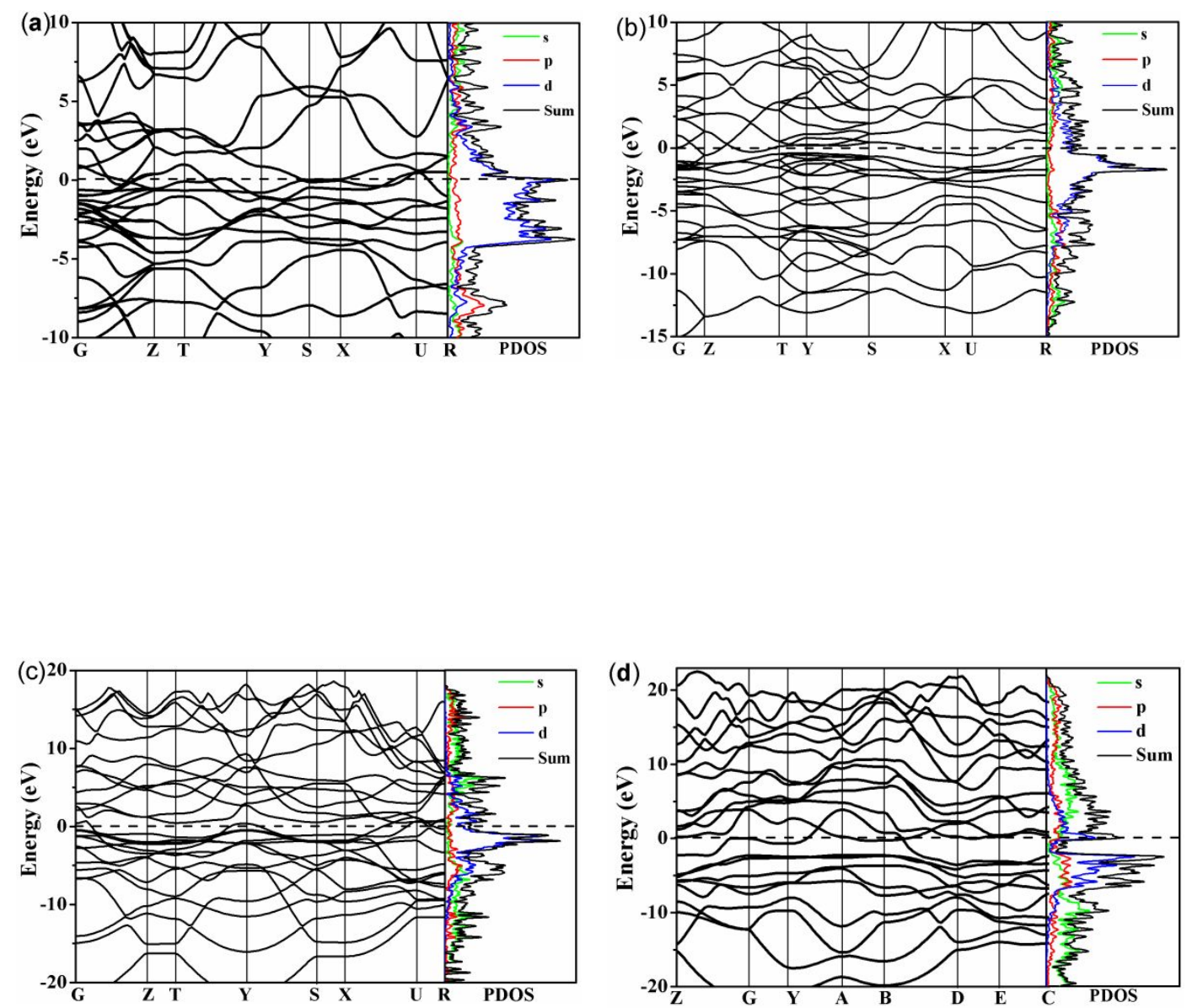

Figure S8. Calculated electronic band structures and partial density of states (PDOSs) of (a) $\mathrm{Immm}-\mathrm{Fe}_{2} \mathrm{SeH}$, (b) $\mathrm{Amm} 2-\mathrm{Fe}_{2} \mathrm{SeH}_{3}$ (c) $\mathrm{Pmmm}-\mathrm{Fe}_{2} \mathrm{SeH}_{6}$ and (d) $\mathrm{Pm}-\mathrm{FeSeH}_{6}$ phases at $150 \mathrm{GPa}$. 

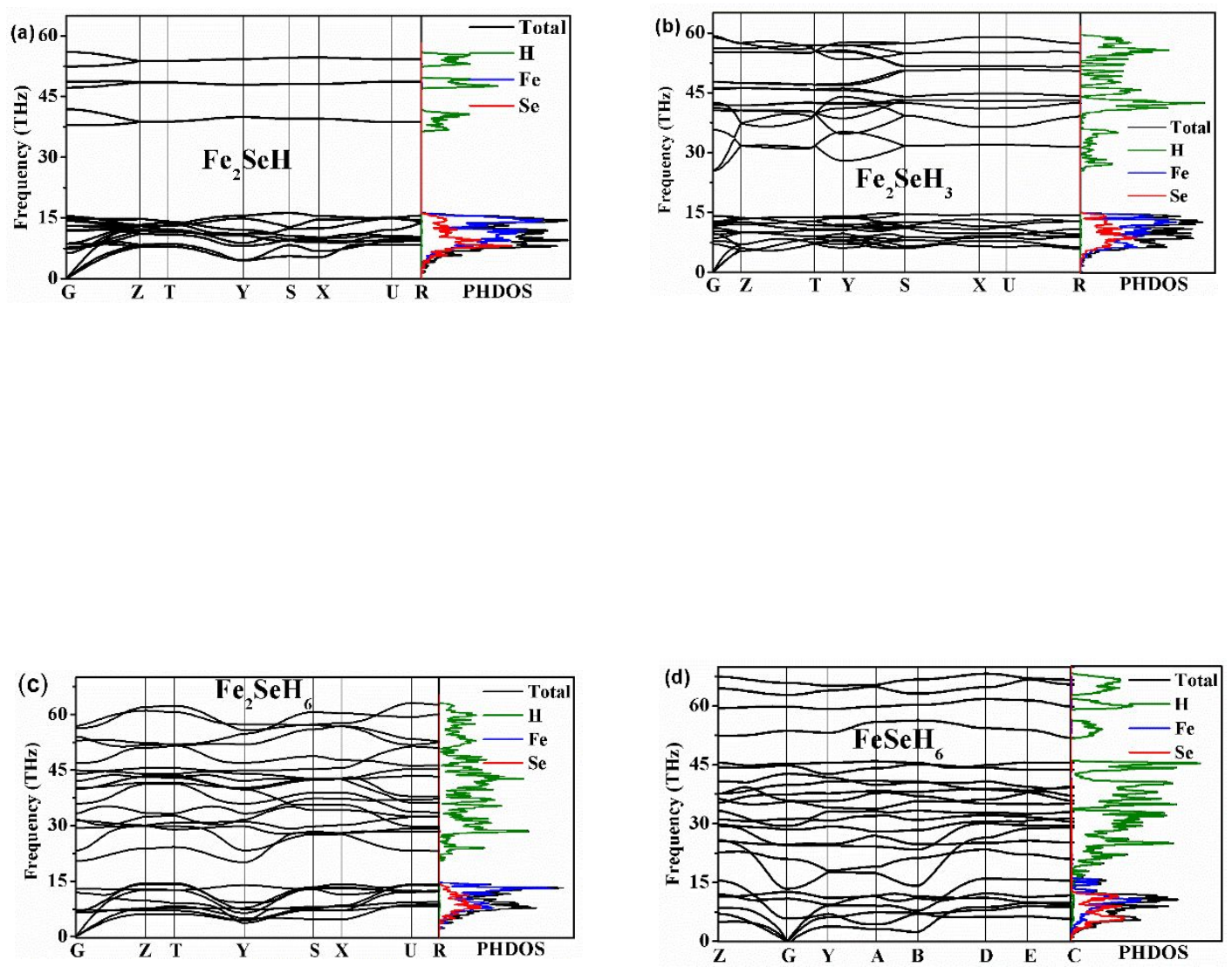

Figure S9. Calculated phonon dispersion curves and phonon density of states (PHDOSs) of (a) Immm- $\mathrm{Fe}_{2} \mathrm{SeH}$, (b) $\mathrm{Amm} 2-\mathrm{Fe}_{2} \mathrm{SeH}_{3}$ (c) $\mathrm{Pmmm}-\mathrm{Fe}_{2} \mathrm{SeH}_{6}$ and (d) $\mathrm{Pm}-\mathrm{FeSeH}_{6}$ phases at $150 \mathrm{GPa}$. 


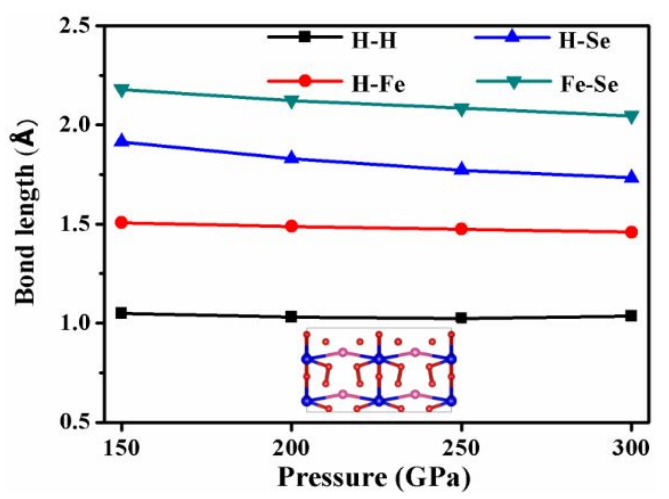

(a)

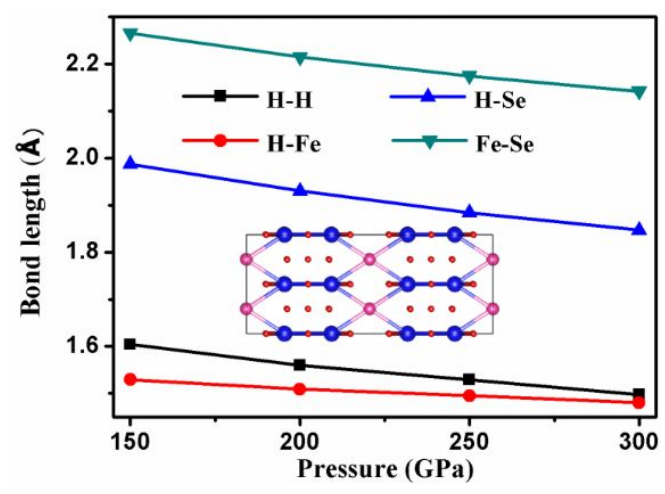

(b)

Figure S10. Variation of the H-H, H-Fe, H-Se and Fe-Se bonds with the increase of pressure from 150 to $300 \mathrm{GPa}$ for (a) $\mathrm{Pm}-\mathrm{FeSeH}_{6}$ and (b) $\mathrm{Pmmm}-\mathrm{Fe}_{2} \mathrm{SeH}_{6}$ phases. 
Table S1. Bader atomic charges of the $\mathrm{C} 2 / \mathrm{m}-\mathrm{FeSeH}$, Immm- $\mathrm{Fe}_{2} \mathrm{SeH}$, $\mathrm{I} 4 / \mathrm{mmm}-\mathrm{Fe}_{2} \mathrm{SeH}_{2}, \quad \mathrm{Amm} 2-\mathrm{Fe}_{2} \mathrm{SeH}_{2}, \quad \mathrm{Amm} 2-\mathrm{Fe}_{2} \mathrm{SeH}_{3}, \quad \mathrm{Pmmm}_{-}-\mathrm{Fe}_{2} \mathrm{SeH}_{6} \quad$ and $\mathrm{Pm}-\mathrm{FeSeH}_{6}$ phases at $150 \mathrm{GPa}$.

\begin{tabular}{|c|c|c|}
\hline Structures & Atoms & Charge \\
\hline \multirow{12}{*}{$\mathrm{C} 2 / \mathrm{m}-\mathrm{FeSeH}$} & $\mathrm{H}$ & -0.23 \\
\hline & $\mathrm{H}$ & -0.23 \\
\hline & $\mathrm{H}$ & -0.17 \\
\hline & $\mathrm{H}$ & -0.18 \\
\hline & $\mathrm{Fe}$ & 0.25 \\
\hline & $\mathrm{Fe}$ & 0.25 \\
\hline & $\mathrm{Fe}$ & 0.29 \\
\hline & $\mathrm{Fe}$ & 0.29 \\
\hline & $\mathrm{Se}$ & -0.10 \\
\hline & $\mathrm{Se}$ & -0.10 \\
\hline & $\mathrm{Se}$ & -0.03 \\
\hline & $\mathrm{Se}$ & -0.04 \\
\hline \multirow{4}{*}{ Immm-Fe ${ }_{2} \mathrm{SeH}$} & $\mathrm{H}$ & -0.18 \\
\hline & $\mathrm{Fe}$ & 0.14 \\
\hline & $\mathrm{Fe}$ & 0.14 \\
\hline & $\mathrm{Se}$ & -0.10 \\
\hline \multirow{2}{*}{$\mathrm{I} 4 / \mathrm{mmm}-\mathrm{Fe}_{2} \mathrm{SeH}_{2}$} & $\mathrm{H}$ & -0.30 \\
\hline & $\mathrm{H}$ & -0.30 \\
\hline
\end{tabular}




\begin{tabular}{|c|c|c|}
\hline & $\mathrm{Fe}$ & 0.33 \\
\hline & $\mathrm{Fe}$ & 0.32 \\
\hline & $\mathrm{Se}$ & -0.06 \\
\hline \multirow{5}{*}{$\mathrm{Amm} 2-\mathrm{Fe}_{2} \mathrm{SeH}_{2}$} & $\mathrm{H}$ & -0.19 \\
\hline & $\mathrm{H}$ & -0.19 \\
\hline & $\mathrm{Fe}$ & 0.21 \\
\hline & $\mathrm{Fe}$ & 0.21 \\
\hline & $\mathrm{Se}$ & -0.02 \\
\hline \multirow{6}{*}{$\mathrm{Amm} 2-\mathrm{Fe}_{2} \mathrm{SeH}_{3}$} & $\mathrm{H}$ & -0.14 \\
\hline & $\mathrm{H}$ & -0.15 \\
\hline & $\mathrm{H}$ & -0.15 \\
\hline & $\mathrm{Fe}$ & 0.24 \\
\hline & $\mathrm{Fe}$ & 0.24 \\
\hline & $\mathrm{Se}$ & -0.03 \\
\hline \multirow{9}{*}{ Pmmm-Fe ${ }_{2} \mathrm{SeH}_{6}$} & $\mathrm{H}$ & -0.16 \\
\hline & $\mathrm{H}$ & -0.08 \\
\hline & $\mathrm{H}$ & -0.07 \\
\hline & $\mathrm{H}$ & -0.17 \\
\hline & $\mathrm{H}$ & -0.14 \\
\hline & $\mathrm{H}$ & -0.13 \\
\hline & $\mathrm{Fe}$ & 0.35 \\
\hline & $\mathrm{Fe}$ & 0.29 \\
\hline & $\mathrm{Se}$ & 0.11 \\
\hline
\end{tabular}




\begin{tabular}{l|c|c}
\hline & $\mathrm{H}$ & -0.02 \\
$\mathrm{H}$ & -0.02 \\
$\mathrm{H}$ & -0.10 \\
$\mathrm{Pm}-\mathrm{FeSeH}_{6}$ & $\mathrm{H}$ & -0.10 \\
$\mathrm{H}$ & -0.01 \\
$\mathrm{H}$ & -0.04 \\
& $\mathrm{Fe}$ & 0.29 \\
& $\mathrm{Se}$ & 0.02 \\
\hline
\end{tabular}


Table S2. Calculated EPC parameter $\lambda$ of the $\mathrm{Pm}-\mathrm{FeSeH}_{6}$ phase at $150 \mathrm{GPa}$ with different sizes of q- and k-points.

\section{k-point}

$8 \times 8 \times 12 \quad 12 \times 12 \times 18 \quad 16 \times 16 \times 24 \quad 24 \times 24 \times 36$ 


\begin{tabular}{|c|c|c|c|c|c|}
\hline & $2 \times 2 \times 3$ & 1.68 & & 1.63 & 1.61 \\
\hline \multirow[t]{3}{*}{ q-point } & $4 \times 4 \times 6$ & 1.24 & & 1.56 & 1.53 \\
\hline & $6 \times 6 \times 9$ & & 1.54 & & \\
\hline & $8 \times 8 \times 12$ & & & 1.51 & \\
\hline
\end{tabular}


Mechanical properties of $\mathrm{C} 2 / \mathrm{m}-\mathrm{FeSeH}, \mathrm{Amm} 2-\mathrm{Fe}_{2} \mathrm{SeH}_{2}$ and $\mathrm{I} / \mathrm{mmm}-\mathrm{Fe}_{2} \mathrm{SeH}_{2}$ phases.

For stable monoclinic, orthorhombic and tetragonal phases, the elastic stiffness constants ${ }^{1}$ should satisfy (monoclinic phase)

$$
\begin{aligned}
& \mathrm{C}_{11}>0, \mathrm{C}_{22}>0, \mathrm{C}_{33}>0 \\
& \mathrm{C}_{44}>0, \mathrm{C}_{55}>0, \mathrm{C}_{66}>0 \\
& \mathrm{C}_{11}+\mathrm{C}_{22}+\mathrm{C}_{33}+2\left(\mathrm{C}_{12}+\mathrm{C}_{13}+\mathrm{C}_{23}\right)>0 \\
& \mathrm{C}_{11}+\mathrm{C}_{22}-2 \mathrm{C}_{12}>0 \\
& \mathrm{C}_{11}+\mathrm{C}_{33}-2 \mathrm{C}_{13}>0 \\
& \left(\mathrm{C}_{22}+\mathrm{C}_{33}-2 \mathrm{C}_{23}\right)>0
\end{aligned}
$$

and (orthorhombic phase)

$$
\begin{aligned}
& \mathrm{C}_{11}>0, \mathrm{C}_{22}>0, \mathrm{C}_{33}>0 \\
& \mathrm{C}_{44}>0, \mathrm{C}_{55}>0, \mathrm{C}_{66}>0 \\
& \mathrm{C}_{11}+\mathrm{C}_{22}+\mathrm{C}_{33}+2\left(\mathrm{C}_{12}+\mathrm{C}_{13}+\mathrm{C}_{23}\right)>0 \\
& \mathrm{C}_{33} \mathrm{C}_{55}-\mathrm{C}_{35}^{2}>0 \\
& \mathrm{C}_{44} \mathrm{C}_{66}-\mathrm{C}_{46}^{2}>0 \\
& \mathrm{C}_{22}+\mathrm{C}_{33}-2 \mathrm{C}_{23}>0
\end{aligned}
$$

and (tetragonal phase)

$$
\begin{aligned}
& \mathrm{C}_{11}>0, \mathrm{C}_{33}>0 \\
& \mathrm{C}_{44}>0, \mathrm{C}_{66}>0 \\
& \mathrm{C}_{11}-\mathrm{C}_{22}>0
\end{aligned}
$$




$$
\begin{aligned}
& \mathrm{C}_{11}+\mathrm{C}_{33}-2 \mathrm{C}_{13}>0 \\
& 2\left(\mathrm{C}_{11}+\mathrm{C}_{22}\right)+\mathrm{C}_{33}+4 \mathrm{C}_{13}>0
\end{aligned}
$$


The elastic constants of the $\mathrm{C} 2 / \mathrm{m}-\mathrm{FeSeH}, \mathrm{Amm} 2-\mathrm{Fe}_{2} \mathrm{SeH}_{2}$ and $\mathrm{I} 4 / \mathrm{mmm}-\mathrm{Fe}_{2} \mathrm{SeH}_{2}$ phases, as listed in Table. S1, satisfy Eqs. (1), (2) and (3), showing that they are mechanically stable.

Table. S3. Elastic constants $\mathrm{Cij}(\mathrm{GPa})$ of the $\mathrm{C} 2 / \mathrm{m}-\mathrm{FeSeH}, \mathrm{Amm} 2-\mathrm{Fe}_{2} \mathrm{SeH}_{2}$ and $\mathrm{I} 4 / \mathrm{mmm}-\mathrm{Fe}_{2} \mathrm{SeH}_{2}$ phases at $150 \mathrm{GPa}$.

\begin{tabular}{ccccccccccccc}
\hline FeSeH & $\mathrm{C} 11$ & $\mathrm{C} 22$ & $\mathrm{C} 33$ & $\mathrm{C} 44$ & $\mathrm{C} 55$ & $\mathrm{C} 66$ & $\mathrm{C} 12$ & $\mathrm{C} 13$ & $\mathrm{C} 23$ & $\mathrm{C} 35$ & $\mathrm{C} 46$ \\
$(\mathrm{C} 2 / \mathrm{m})$ & 870.58 & 919.08 & 927.50 & 246.52 & 280.31 & 267.82 & 553.06 & 527.17 & 489.61 & -23.30 & -3.64 \\
\hline $\mathrm{Fe}_{2} \mathrm{SeH}_{2}$ & $\mathrm{C} 11$ & $\mathrm{C} 22$ & $\mathrm{C} 33$ & $\mathrm{C} 44$ & $\mathrm{C} 55$ & $\mathrm{C} 66$ & $\mathrm{C} 12$ & $\mathrm{C} 13$ & $\mathrm{C} 23$ & \\
$(\mathrm{Amm} 2)$ & 881.77 & 1062.83 & 956.95 & 223.33 & 218.05 & 250.79 & 582.74 & 485.38 & 555.65 & \\
\hline $\mathrm{Fe}_{2} \mathrm{SeH}_{2}$ & $\mathrm{C} 11$ & $\mathrm{C} 33$ & $\mathrm{C} 44$ & $\mathrm{C} 66$ & $\mathrm{C} 12$ & $\mathrm{C} 13$ & & & & \\
$(\mathrm{I} 4 / \mathrm{mmm})$ & 792.15 & 850.69 & 108.26 & 158.48 & 593.01 & 679.71 & & & & & & \\
\hline
\end{tabular}

1. Wu, Z. J.; Zhao, E. J.; Xiang, H. P.; Hao, X. F.; Liu, X. J.; Meng, J. Crystal Structures and Elastic Properties of Superhard $\mathrm{IrN}_{2}$ and $\mathrm{IrN}_{3}$ from First Principles. Phys. Rev. B. 2007, 76 (5), 054115. 
Table. S4. Lattice parameters and atomic coordinates of the $\mathrm{C} 2 / \mathrm{m}-\mathrm{FeSeH}, \mathrm{Pm}_{-} \mathrm{FeSeH}$ Immm- $\mathrm{Fe}_{2} \mathrm{SeH}, \quad \mathrm{Amm} 2-\mathrm{Fe}_{2} \mathrm{SeH}_{2}, \quad \mathrm{I} 4 / \mathrm{mmm}-\mathrm{Fe}_{2} \mathrm{SeH}_{2}, \quad \mathrm{Amm} 2-\mathrm{Fe}_{2} \mathrm{SeH}_{3}, \quad$ and Pmmm- $\mathrm{Fe}_{2} \mathrm{SeH}_{6}$ phases in fractional coordinates.

\begin{tabular}{|c|c|c|c|c|c|c|}
\hline \multirow{2}{*}{ phase } & \multirow{2}{*}{$\begin{array}{l}\text { Pressure } \\
(\mathrm{GPa})\end{array}$} & \multirow{2}{*}{$\begin{array}{c}\text { Lattice } \\
\text { parameters }\end{array}$} & \multirow{2}{*}{ Atoms } & \multicolumn{3}{|c|}{ Atomic coordinates } \\
\hline & & & & & ctional & \\
\hline \multirow{4}{*}{$\mathrm{FeSeH}$} & \multirow{6}{*}{150} & $\mathrm{a}=4.89$ & $\mathrm{H}(1)$ & 0.89 & 0.89 & 0.46 \\
\hline & & $\mathrm{b}=4.89$ & $\mathrm{H}(2)$ & 0.46 & 0.46 & 0.39 \\
\hline & & $\mathrm{c}=8.22$ & $\mathrm{Fe}(3)$ & 0.30 & 0.30 & 0.43 \\
\hline & & $\alpha=129.8$ & $\mathrm{Fe}(4)$ & 0.91 & 0.91 & 0.27 \\
\hline \multirow{2}{*}{$(\mathrm{C} 2 / \mathrm{m})$} & & $\beta=129.8$ & $\operatorname{Se}(5)$ & 0.57 & 0.57 & 0.24 \\
\hline & & $\gamma=34.4$ & $\operatorname{Se}(6)$ & 0.18 & 0.18 & 0.07 \\
\hline \multirow{6}{*}{$\begin{array}{c}\mathrm{FeSeH}_{6} \\
(\mathrm{Pm})\end{array}$} & \multirow{6}{*}{150} & $\mathrm{a}=3.49$ & $\mathrm{H}(1)$ & 0.09 & 0.23 & 0.68 \\
\hline & & $b=3.86$ & $\mathrm{H}(2)$ & 0.20 & 0.29 & 0.08 \\
\hline & & $\mathrm{c}=2.37$ & $\mathrm{H}(3)$ & 0.56 & 0.00 & 0.86 \\
\hline & & $\alpha=90$ & $\mathrm{H}(4)$ & 0.44 & 0.00 & 0.32 \\
\hline & & $\beta=72.3$ & $\mathrm{Fe}(5)$ & 0.89 & 0.00 & 0.26 \\
\hline & & $\gamma=90$ & $\operatorname{Se}(6)$ & 0.59 & 0.50 & 0.43 \\
\hline \multirow{6}{*}{$\begin{array}{l}\mathrm{Fe}_{2} \mathrm{SeH} \\
(\mathrm{Immm})\end{array}$} & \multirow{6}{*}{150} & $a=6.04$ & $\mathrm{H}(1)$ & 0.00 & 0.00 & 0.00 \\
\hline & & $\mathrm{b}=3.80$ & $\mathrm{Fe}(2)$ & 0.30 & 0.50 & 0.00 \\
\hline & & $\mathrm{c}=2.42$ & & & & \\
\hline & & $\alpha=90$ & $\operatorname{Se}(3)$ & 0.00 & 0.50 & 0.50 \\
\hline & & $\beta=90$ & & & & \\
\hline & & $\gamma=90$ & & & & \\
\hline
\end{tabular}




\begin{tabular}{|c|c|c|c|c|c|c|}
\hline & & & $\mathrm{H}(1)$ & 0.50 & 0.23 & 0.89 \\
\hline & & & $\mathrm{H}(2)$ & 0.50 & 0.73 & 0.39 \\
\hline & & $a=2.66$ & $\mathrm{H}(3)$ & 0.50 & 0.77 & 0.89 \\
\hline & & $b=3.20$ & $\mathrm{H}(4)$ & 0.50 & 0.27 & 0.39 \\
\hline \multicolumn{7}{|l|}{$\mathrm{Fe}_{2} \mathrm{SeH}_{2}$} \\
\hline & & $\mathrm{c}=7.04$ & $\mathrm{Fe}(5)$ & 0.00 & 0.00 & 0.37 \\
\hline \multirow[t]{9}{*}{$(\mathrm{Amm} 2)$} & 150 & & & & & \\
\hline & & $\alpha=90$ & $\mathrm{Fe}(6)$ & 0.00 & 0.50 & 0.87 \\
\hline & & $\beta=90$ & $\mathrm{Fe}(7)$ & 0.50 & 0.00 & 0.08 \\
\hline & & $\gamma=90$ & $\mathrm{Fe}(8)$ & 0.50 & 0.50 & 0.58 \\
\hline & & & $\mathrm{Se}(9)$ & 0.00 & 0.50 & 0.17 \\
\hline & & & $\operatorname{Se}(10)$ & 0.00 & 0.00 & 0.67 \\
\hline & & $\mathrm{a}=2.61$ & $\mathrm{H}(1)$ & 0.50 & 0.00 & 0.75 \\
\hline & & $b=2.61$ & & & & \\
\hline & & & $\mathrm{Fe}(2)$ & 0.50 & 0.50 & 0.36 \\
\hline \multirow[t]{2}{*}{$\mathrm{Fe}_{2} \mathrm{SeH}_{2}$} & & $c=8.72$ & & & & \\
\hline & 150 & & & & & \\
\hline \multirow[t]{5}{*}{$(\mathrm{I} 4 / \mathrm{mmm})$} & & $\alpha=90$ & & & & \\
\hline & & $\beta=90$ & $\mathrm{Se}(3)$ & 0.00 & 0.00 & 0.50 \\
\hline & & $\gamma=90$ & & & & \\
\hline & & $a=2.70$ & $\mathrm{H}(1)$ & 0.50 & 0.50 & 0.44 \\
\hline & & $b=3.23$ & $\mathrm{H}(2)$ & 0.00 & 0.26 & 0.51 \\
\hline \multirow[t]{2}{*}{$\mathrm{Fe}_{2} \mathrm{SeH}_{3}$} & & $c=7.22$ & $\mathrm{Fe}(3)$ & 0.00 & 0.50 & 0.31 \\
\hline & 150 & & & & & \\
\hline \multirow[t]{4}{*}{$(\mathrm{Amm} 2)$} & & $\alpha=90$ & $\mathrm{Fe}(4)$ & 0.50 & 0.00 & 0.52 \\
\hline & & $\beta=90$ & & & & \\
\hline & & & $\mathrm{Se}(5)$ & 0.50 & 0.00 & 0.22 \\
\hline & & $\gamma=90$ & & & & \\
\hline \multirow[t]{2}{*}{$\mathrm{Fe}_{2} \mathrm{SeH}_{6}$} & & $a=6.15$ & $\mathrm{H}(1)$ & 0.84 & 0.00 & 0.00 \\
\hline & 150 & & & & & \\
\hline (Pmmm) & & $b=2.47$ & $\mathrm{H}(2)$ & 0.33 & 0.50 & 0.00 \\
\hline
\end{tabular}




\begin{tabular}{cccccc}
\hline $\mathrm{c}=2.43$ & $\mathrm{H}(3)$ & 0.50 & 0.50 & 0.50 \\
$\alpha=90$ & $\mathrm{H}(4)$ & 0.50 & 0.00 & 0.00 \\
$\beta=90$ & $\mathrm{Fe}(5)$ & 0.31 & 0.00 & 0.50 \\
& $\gamma=90$ & $\mathrm{Se}(6)$ & 0.00 & 0.50 & 0.50 \\
\hline
\end{tabular}

